\section{An evaluation of remote communication versus face-to- face in clinical dental education}

\author{
N. Martin, ${ }^{1}$ O. Martínez Lazalde, ${ }_{1}^{2}$ C. Stokes ${ }^{3}$ and D. Romano ${ }^{4}$
}

IN BRIEF

- Physical face-to-face learning remains a very effective communication modality for clinical case discussions between a learner and an expert.

- Internet-based clinical case discussions enable a more relaxed environment than physical face-to-face encounters, which benefits the learning process.

- 3D-supported internet communication is a promising technology requiring prior training to be an effective user-interface.

Distance learning and internet-based delivery of educational content are becoming very popular as an alternative to real face-to-face delivery. Clinical-based discussions still remain greatly face-to-face despite the advancement of remote communication and internet sharing technology. In this study we have compared three communication modalities between a learner and educator: audio and video using voice over internet protocol (VoIP) alone [AV]; audio and video VoIP with the addition of a three dimensional virtual artefact [AV3D] and physical face-to-face [FTF]. Clinical case discussions based on fictitious patients were held between a 'learner' and an 'expert' using the three communication modalities. The learner presented a clinical scenario to the experts, with the aid of a prop (partially dentate cast, digitised for AV3D), to obtain advice on the management of the clinical case. Each communication modality was tested in timed exercises in a random order among one of three experts (senior clinical restorative staff) and a learner (from a cohort of 15 senior clinical undergraduate students) all from the School of Clinical Dentistry, University of Sheffield. All learners and experts used each communication modality in turn with no prior training. Video recording and structured analysis were used to ascertain learner behaviour and levels of interactivity. Evaluation questionnaires were completed by experts and learners immediately after the experiment to ascertain effectiveness of information exchange and barriers/facilitators to communication. The video recordings showed that students were more relaxed with AV and AV3D than FFF ( $p=0.01)$. The evaluation questionnaires showed that students felt they could provide $(p=0.03)$ and obtain $(p=0.003)$ more information using the FTF modality, followed by $A V$ and then AV3D. Experts also ranked FTF better than AV and AV3D for providing $(p=0.012)$ and obtaining $(p=0)$ information to/from the expert. Physical face-to-face learning is a more effective communication modality for clinical case-based discussions between a learner and an expert. Remote, internet-based discussions enable a more relaxed discussion environment. The effectiveness of 3D supported internet-based communication is dependent upon a robust and simple to use interface, along with some prior training.

\section{INTRODUCTION}

The provision of postgraduate education in medicine and dentistry is critical to maintaining standards and professional revalidation in the UK. Revalidation is the process by which doctors and dentists have to demonstrate on a regular basis to the General Medical Council and General Dental Council respectively that they remain up to date and fit to practice. It is the responsibility of individuals and

\footnotetext{
"University of Sheffield, Academic Unit of Restorative Dentistry, School of Clinical Dentistry, Claremont Crescent, Sheffield, S10 2TA; ${ }^{2}$ Computer Science, Regent Court, 211 Portobello, Sheffield, S1 4DP; ${ }^{3}$ University of Sheffield, Sheffield, S10 2TA; ${ }^{4}$ Computer Science, Regent Court, 211 Portobello, Sheffield, S1 4DP *Correspondence to: Dr Nicolas Martin Email:n.martin@sheffield.ac.uk
}

\section{Refereed Paper}

Accepted 15 December 2011

DOI: 10.1038/sj.bdj.2012.226

${ }^{\circ}$ British Dental Journal 2012; 212: 277-282 the professional organisations to which they belong to ensure that the appropriate training and education is provided and received.

Distance learning is increasing in popularity with the medical/dental profession as it can be delivered by the teacher and received by the learner with great convenience to both parties. Teleconferencing and more recently internet-based tutorials (webinars) are also becoming increasingly popular. ${ }^{1}$ A drawback of these distance learning modalities is that they are largely uni-directional in the delivery of learning, although it is accepted that learner participation does occur in the form of Q\&A sessions. One important aspect of effective postgraduate education relies on the ability to have a dynamic two-way conversation/ discussion centred on a clinical scenario case-based clinical discussions. This may be from a purely academic perspective as a learning exercise or with direct practical applicability to resolve a true clinical problem where the learner is seeking the specialist opinion of an expert.

Case-based clinical discussions often require the constant exchange of large clinical data sets between the learner and the expert, so that all the diverse, key elements of clinical information are understood and used to enable the establishment of a diagnosis, prognosis and appropriate treatment strategy. Traditionally, such educational exchanges are made in a face-toface setting with all the clinical data at hand, which may also include the physical presence of the patient. These learning episodes are very inflexible from a logistical planning point of view, with a requirement for a common venue, date and time, as well as the need to co-ordinate all 
the relevant information for the exercise to take place. This also makes them a noncost-effective educational delivery mode.

The authors postulate that case-based clinical discussions, either as an educational exercise or for direct patient management, will benefit from the use of super-fast broadband connectivity to improve the quality and cost-effectiveness of the service by enabling remote consultations to take place with a rapid and secure exchange of large clinical data sets. In the UK, the government wants the UK to have the best super-fast broadband network in Europe by 2015, with remote educational services being one of the specific benefits. ${ }^{2}$

Further potential advantages of superfast internet-based clinical discussions are that they may enable greater spontaneity of engagement from the learner by not being in the physical, sometimes intimidating, presence of the 'expert'. This may lead to a better informed learning outcome resulting from a more relaxed interaction. Extra clarity can be gained from an expert's comments on a written report when the material can be downloaded instead of posted and the logistics of the consultation are easier to manage.

Within healthcare, dentistry is a selfcontained medical discipline that lends itself to the exploration of remote clinical education. In this paper we report a study to identify the opportunities and challenges associated with the delivery of remote clinical education in dentistry using super-fast broadband connectivity.

The use of the internet for the delivery of medical and dental education has escalated rapidly and is seeing an exponential growth. ${ }^{3}$ Distance learning relies on the internet and information and communication technology (ICT) and is having an important impact on education in dentistry. ${ }^{3-5}$ Feeney et al. and Eaton, in a review of the current ICT technologies used in dental education, found that students were better able to manage their time and location of learning. ${ }^{1,6}$ Also, students found greater ease in having more frequent and easy collaboration and contact with teaching staff and fellow students. ${ }^{1,6}$ Successful interaction with people via technology depends not just on personal relationships, but also on the understanding and the ability of teachers and students on how to best use computers and communications equipment effectively. ${ }^{7}$ A further benefit for dental education, highlighted by Reynolds and Eaton, is that students can save lectures so that people unable to see or attend can watch them in their own time and also use them for revision purposes. ${ }^{8}$

With the speed improvement of internet devices, broadband-based videoconferencing is becoming a popular option for distance-learning. Now it is possible to communicate freely using the voice over internet protocol (VoIP). There are some free VoIP programs, Skype currently being the most popular (http://www.skype.com/ intl/en-gb/home). This program enables videoconference calls between a dentist and an expert and allows its potential use in dental practices to be explored. ${ }^{9,10}$

The videoconference's effectiveness and users' acceptance has been investigated. Reynolds and Mason argue that teaching dentistry sessions by videoconferencing are as effective as traditional face-to-face lectures. ${ }^{11}$ Kenneth et al. evaluated the effectiveness and acceptability of three methods of interaction (videoconference, audio and text chat) in a seminar scenario with a discussion between an instructor and students. ${ }^{12}$ Videoconferencing received the highest ratings and was never cited as the least favourite method of interaction. Audio interaction was in second place and text in third. In a study on orthodontic consultants' views about providing advice using electronic means, ${ }^{5,10}$ it was found that 58\% were interested in using the new technology clinically, with $70 \%$ in favour of doing further research into the topic.

A challenge for remote clinical consultations using internet connectivity in medicine and dentistry is the need to exchange visual 3D information. The ability to convey this information to both parties in a speedy manner with full interactivity of the data by both parties is key to the success of this educational modality.

The hypothesis for this study is that 3D supported remote digital communication in clinical dentistry is an effective alternative to face-to-face communication between a learner and an expert. The aim was to assess the barriers and relative merits of using a VoIP alone or with the addition of a three dimensional virtual artefact to aid discussion for remote dental education. These are compared to a traditional educational face-to-face setting between a learner and educator.

\section{METHODS}

A clinical case discussion for a fictitious patient between a learner (clinical dental undergraduate student) and an expert (senior clinical academic) was held using three different communication modalities: face-to-face (FTF); computer supported audio and video communication (AV) and a virtual environment supporting video and audio communication alongside the sharing of a three-dimensional artefact to aid discussion (AV3D). The structure of the discussion was such that the learners presented a clinical scenario, with the aid of a prop (partially dentate cast) to the experts to obtain advice on the management of the clinical case being prepared and the most appropriate design for a removable partial denture. Three different scenarios were used, one for communication modality. A dialogue was thus established through which the experts sought further information from the learner and provided them with an appropriate management strategy for the fictitious patient. Each communication modality was tested in exercises in a random order between an expert (one of three: A, B and C) and a student, from a cohort of 15 senior clinical dental under-graduate students. The experts are members of the Academic Unit of Restorative Dentistry from the School of Clinical Dentistry of the University of Sheffield. The students were senior clinical dental undergraduate students in the fourth year of the same school split into two groups: group one consisting of six students (two male and five female) and group two consisting of eight students (two male and six female).

All learners took part in each of the three communication modalities. Both the learners and experts carried out a calibration exercise before the experiment to establish uniformity of information and data relayed between them. The learners completed a pre-test assessment exercise that ensured that they met the requirements to take part in the experiment, these being:

- An appropriate level of knowledge and competency to enable an appropriate discussion of the clinical scenario

- Ability to manipulate a 3D object on a computer monitor with the PC mouse. 


\section{D remote interaction tool (Fig. 1)}

The materials used on the experiment were:

1. Three different solid acrylic replica models of simulated partially dentate human arch, one of which was digitised using a 3D scanner (NextEngine 3D Laser Scanner, running ScanStudio HD Pro)

2. A 3D remote interaction tool that shows the digitised dental model (Fig. 1). The same model is duplicated on the left and right side of the screen and visible to both individuals simultaneously. The learner and the expert have control of a model as indicated on the screen. Both individuals can rotate and draw points on the 3D model to aid the discussion

3. A fictitious clinical data set for each partially dentate case (age, gender, presenting complaint, relevant dental and medical history, dental/restorative chart and special investigations).

The modality exercises are defined as follows:

1. Face-to-face (FTF): the learner used the clinical data set and matching solid replica model (Clinical scenario 1) to engage in a discussion with the expert sitting across a table. Both the expert and the learner had direct access to the dental model. The learner drew the suggested denture design on a prescription card.

2. Audio and video communication (AV): the learner used the clinical data set and matching solid replica model (Clinical scenario 2) to engage in a remote discussion with the expert via a VoIP program (Skype) using a microphone and a webcam. Only the learner had direct access to the dental model, the expert could only see it via the webcam and had to give instructions to the learner to manipulate the model in order to view this. The learner drew the suggested denture design on a prescription card

3. Audio, video and 3D communication (AV3D): the learner used the clinical data set and the digitised replica model (Clinical scenario 3) to engage in a remote discussion with the expert. They had the same level of

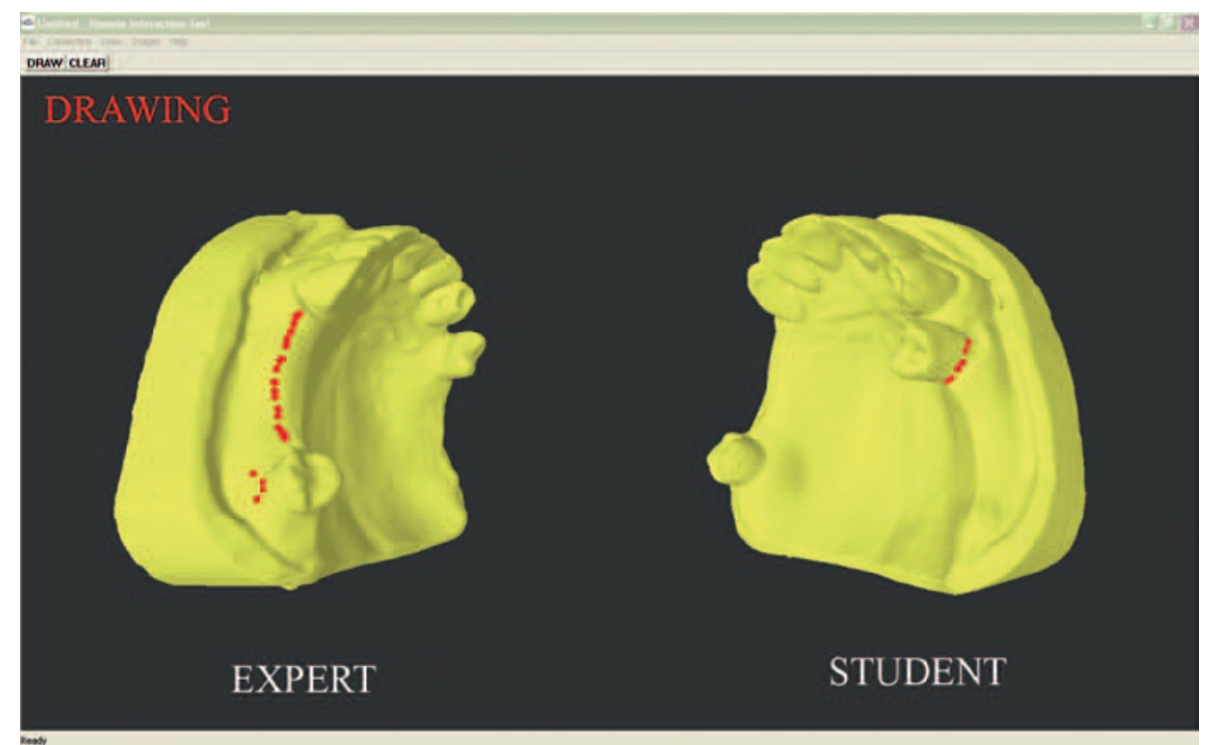

Fig. $13 D$ remote interaction tool

connectivity as the AV modality (VoIP program - Skype) and in addition, the learner and the expert could visualise, manipulate and draw on the digitised 3D model. This way, both the expert and the learner had direct access to the dental model. The learner drew the suggested denture design on a prescription card.

The university's intranet high-speed fibre optic network was used to simulate high speed commercial broadband connectivity. Each computer was connected locally at 100 Mbit and used a shared 1-2 Gbit service to interconnect them. Local testing showed the connection to the wider internet (required for Skype) to average $60 \mathrm{Mbps}$ download and $20 \mathrm{Mbps}$ upload speeds.

The experiments were assessed qualitatively and quantitatively performing:

- Video analysis of the interaction of people and artefacts (whether real or computer based) according to the activity theory ${ }^{14}$

- Statistical evaluation of students and experts' responses to questionnaire immediately after the experience.

The experiment interactions were videorecorded in full to enable an assessment of the behavioural interaction between learner and expert and the artefacts (the real and 3D cast and the software). A subsequent visual analysis was undertaken of these recordings at three different stages (beginning, middle and end of the experiment). To reduce subjectivity and bias, the visual analysis was carried out by the four evaluators independently, where agreement on the assigned score was reached by voting. According to Bødker ${ }^{13}$ focus shifts and breakdowns are instrumental for analysing interactions in humancomputer interaction. Activities never take place in isolation and artefacts are mediators allowing people to focus their attention. An artefact works well in our activity if it helps to focus attention, badly if it does not. Consequently, breakdowns and focus shifts are useful indicators to understand whether an artefact and computer application does or does not mediate an activity, in our case the learner-expert communication.

In the video analysis, the following parameters were examined at these key stages:

\section{Learner non-verbal behaviour}

- Learner's feelings (apprehensive or relaxed) and the learner's situational knowledge (confused or demonstrating a good understanding)

- The learners' eye contact or focus (appropriately shared between cast and expert, or primarily at the cast or avoiding eye contact with the expert)

- The body language of the learner and the expert (who was more empathetic and therefore facilitating the discussion).

\section{General interaction focus factors}

- Who was leading the conversation most of the time? 
- Who was manipulating the cast or the 3D model most of the time?

- Who was doing most of the pointing at the cast or 3D model?

Furthermore an evaluation questionnaire was issued to both the expert and the learner immediately after the experiment. The following parameters were evaluated:

- Ability to obtain sufficient information from the discussion ('obtain' score)

- Ability to provide sufficient information for the discussion ('provide' score)

- Barriers to communication ('barrier' score)

- Best and worst aspects of the particular communication mode being tested.

All the learners in each group engaged with each expert using a different modality as depicted in Table 1. A schedule for the logistics of the experiment was created ensuring each student performed the three different modalities.

\section{Experiment design}

A number of rooms were used for the experiment: five experiment rooms and one control room from which the logistics of the study were managed. Of the five experiment rooms, two were located in a remote area of the building (rooms 4 and 5), from which the experts were conducting the remote interaction (Table 1).

Each room was set up in a similar manner, with a clinical data sheet and dental casts in each of the rooms. The students were asked to arrive ten minutes before at the control room so they could have the study explained to them. As there were three experts and three modalities the students performed the experiment in groups of three.

1. Each student in the group of three went to different rooms according to the pre-arranged schedule

2. Student and expert spent two minutes doing a baseline test. The students were given a simple exercise where they had to describe a partially dentate replica model to the expert. Quality of output, time spent and type of questions asked were assessed to establish a baseline of communication between that particular learner and the expert

\begin{tabular}{|c|c|c|}
\hline Modality & Group 1 & Group 2 \\
\hline FTF (room 1) & Expert A (in room 1) & Expert $C$ (in room 1$)$ \\
\hline $\mathrm{AV}$ (room 2) & Expert B (in room 4) & Expert A (in room 4) \\
\hline AV3D (room 3) & Expert C (in room 5) & Expert B (in room 5) \\
\hline
\end{tabular}

\begin{tabular}{|c|c|c|c|c|c|c|c|c|c|c|}
\hline \multirow{2}{*}{ Learner } & \multicolumn{2}{|l|}{ AV3D } & \multicolumn{2}{|l|}{$\mathrm{AV}$} & \multicolumn{2}{|l|}{ FTF } & \multicolumn{4}{|c|}{ Friedman test } \\
\hline & Mean & Std dev & Mean & Std dev & Mean & Std dev & $F$ & $a$ & $p$ & Significant \\
\hline Provide & 4.130 & 0.92 & 4.270 & 0.59 & 4.730 & 0.590 & 7.000 & 0.05 & 0.030 & Yes \\
\hline Obtain & 4.330 & 0.62 & 4.400 & 0.51 & 4.930 & 0.260 & 11.353 & 0.05 & 0.050 & Yes \\
\hline Barrier? & 1.800 & 1.660 & 2.870 & 2.070 & 4.730 & 1.030 & 16.909 & 0.05 & 0.000 & Yes \\
\hline
\end{tabular}

Table 3 Results from expert evaluations

\begin{tabular}{|c|c|c|c|c|c|c|}
\hline \multirow{2}{*}{ Expert } & \multicolumn{2}{|l|}{ AV3D } & \multicolumn{2}{|l|}{ AV } & \multicolumn{2}{|l|}{ FTF } \\
\hline & Mean & Std dev & Mean & Std dev & Mean & Std dev \\
\hline Provide & 3.470 & 0.920 & 4.000 & 1.110 & 4.400 & 0.630 \\
\hline Obtain & 3.730 & 0.880 & 4.000 & 1.040 & 4.800 & 0.410 \\
\hline Barrier? & 1.530 & 1.410 & 3.290 & 2.050 & 3.290 & 2.050 \\
\hline
\end{tabular}

Table 4 Results from video analysis

\begin{tabular}{|c|c|c|c|c|c|c|c|}
\hline & $A V 3 D$ & $A V$ & FTF & & Friedman & & \\
\hline Learner Feeling & \multicolumn{3}{|c|}{ Relaxed(5) Apprehensive(1) } & $\mathrm{F}$ & $\alpha$ & $p$ & Significant \\
\hline Beginning & 5.000 & 5.000 & 2.670 & 14.000 & 0.05 & 0.001 & Yes \\
\hline Middle & 5.000 & 5.000 & 2.670 & 14.000 & 0.05 & 0.001 & Yes \\
\hline End & 4.670 & 5.000 & 3.000 & 10.330 & 0.05 & 0.006 & Yes \\
\hline Knowledge & \multicolumn{3}{|c|}{ Understanding(5) Not U(1) } & & & & \\
\hline Beginning & 4.670 & 5.000 & 4.670 & 1.000 & 0.05 & 0.607 & No \\
\hline Middle & 4.670 & 4.670 & 5.000 & 1.000 & 0.05 & 0.607 & No \\
\hline End & 5.000 & 5.000 & 5.000 & - & 0.05 & - & - \\
\hline Empathy & \multicolumn{3}{|c|}{ Learner(5) Both(3) Expert(1) } & & & & \\
\hline Beginning & 2.330 & 3.330 & 2.330 & 8.000 & 0.05 & 0.018 & Yes \\
\hline Middle & 3.000 & 3.670 & 2.330 & 8.700 & 0.05 & 0.013 & Yes \\
\hline End & 3.000 & 3.330 & 2.830 & 2.330 & 0.05 & 0.311 & No \\
\hline Eye contact & \multicolumn{3}{|c|}{ Appropriate(5) Cast(3) Away(1) } & & & & \\
\hline Beginning & 5.000 & 5.000 & 5.000 & - & 0.05 & - & - \\
\hline Middle & 5.000 & 5.000 & 4.500 & 6.000 & 0.10 & 0.050 & Yes \\
\hline End & 5.000 & 5.000 & 4.330 & 8.000 & 0.05 & 0.018 & Yes \\
\hline General & \multicolumn{3}{|c|}{ Learner(5) Both(3) Expert(1) } & & & & \\
\hline Leading & 1.500 & 1.170 & 1.000 & 4.670 & 0.10 & 0.097 & Yes \\
\hline Manipulation & 2.830 & 5.000 & 4.330 & 18.670 & 0.05 & 0.000 & Yes \\
\hline Pointing & 2.830 & 5.000 & 4.500 & 17.640 & 0.05 & 0.000 & Yes \\
\hline
\end{tabular}


3. Each student and expert performed an experiment modality according to the experiment schedule for around ten minutes

4. While doing the experiment and at the end, each expert filled in an expert evaluation form

5. The students went to the control room to fill in a learner evaluation form

6. The students repeated points one to five for the other two modalities they had not done.

This procedure was done until all of the students of group one performed all three modalities. Then the experts changed room or cubicle to perform a different modality with group two. Group two followed the same procedure as group one.

Ethical approval was obtained from the University of Sheffield Research Ethics Committee (School of Clinical Dentistry) for the research described.

\section{ANALYSIS OF RESULTS}

\section{Questionnaire results}

\section{Results from learner and expert} evaluation (Tables 2 and 3 )

The responses were assigned a number from one (disagree) to five (agree) for the 'provide' and 'obtain' questions. In the case of the 'no barriers' question the scoring was inverted and a value of one was assigned to 'yes, there are barriers to communication' and five to 'no, there are no barriers to communication'. The table shows in each cell the average for each group in the evaluation. In the second column, results for the AV3D modality are shown, on the third for the AV modality and on the fourth for the FTF. The test scores were subjected to the Friedman test to detect differences across the multiple experimental events.

In Table 2 it can be observed that the Friedman test applied to the modality results according to the learners' evaluation is significant ( $\alpha=0.05$ ) for ability to provide information ( $p=0.03$ ), for ability to obtain information ( $p=0.003$ ) and for learners not experiencing barriers to communication ( $\mathrm{p}=0$ ). Thus, according to the learners' evaluation, FTF modality is the best to provide and obtain, with no barriers of communication. It is followed by $\mathrm{AV}$ and then AV3D. Similar results were obtained from the experts' evaluation. This can be observed in the significant result of the Friedman test applied to the modality results $(\alpha=0.05)$ for ability to provide information $(\mathrm{p}=0.012)$ and for ability to obtain information $(p=0)$. For learners not experiencing barriers on communication results were significant $(\alpha=0.1, p=0.082)$.

As each group performed each modality with different experts, the difference between experts could have an effect on the results of the modality. The Wilcoxon test was applied, fixing modality across the experts, and the results ( $\alpha=0.05$ ) for AV3D ( $p=0.165)$, for AV $(p=0.295)$ and for FTF ( $p=0.336)$ indicate that there is no significant difference among experts. The order in which each learner performed the modalities was random and in some cases there was not enough data to study whether there was an order effect. When comparing five samples performing a modality first and five performing at the end, the results were statistically not significant.

\section{Video results}

\section{Results from video analysis (Table 4)}

Each video was firstly analysed independently by the research investigators and consensually afterwards to reach agreement. A number between one and five was assigned for each parameter observed. The table shows in each cell the average across videos. In the second column, results for the AV3D modality are shown, on the third for the AV modality and on the fourth for FTF. From the fifth to the eight columns values related to the Friedman test are expressed.

For the parameter 'learner feeling' significant differences $(\alpha=0.05)$ exist at the beginning $(p=0.001)$, middle $(p=0.001)$ and end of each exercise $(p=0.006)$. Learners were more apprehensive in FTF.

For the parameter 'empathy' significant differences $(\alpha=0.05)$ are noted at the beginning ( $p=0.018)$ and at the end $(p=0.013)$. It can be concluded that learners where more empathetic in AV3D and AV than in FTF.

For the parameter 'eye contact' significant differences ( $\alpha=0.1, p=0.05$ ) are noted at the middle and at the end $(\alpha=0.05, p=0,018)$. There was more eye contact in AV3D and AV than FTF.
For the 'general factors' parameter significant differences were noted for leading conversation ( $\alpha=0.01, p=0.097$ ), for manipulation ( $\alpha=0.05, p=0$ ) and pointing $(\alpha=0.05, p=0)$. The rest of the results are not significant.

\section{DISCUSSION}

Physical face-to-face learning was perceived a more effective communication modality for clinical case-based discussions between a learner and an expert. This was something expected as it is the natural method of communication and considered as the gold standard for clinical consultations.

Remote, internet-based discussions enable a more relaxed discussion environment. These two modalities offer interesting advantages such as learners being less apprehensive, unlike in FTF where they may get the impression the consultation is an examination. Good eye contact was observed in the three modalities, but less in FTF. Although at the beginning people in $\mathrm{AV}$ were more empathetic, by the end of the AV3D session they were better in this modality. In the three modalities the expert mainly led the conversation but in FTF this was more evident as the difference in status was exposed and as a result the expert kept asking and the learner replying. In AV and AV3D this status difference was not exposed and as a result the learners were relaxed, they behaved as if they were speaking to a friend and observations showed they were comfortable with the camera. Some of the subjects forgot that they were speaking in front of a webcam, while some of them were very aware of the video link so they actively used that to help with the information gathering exercise. The conversation was more equal, with equal amount of dialogue as they did not feel inhibited by the presence of the camera or the expert. Overall, this study suggests that when using AV and AV3D students felt they had more control of the environment. Although we should consider that a subject's recall of their experience as reported in the post sessions questionnaire could often be biased by their perception and does not reflect the actual events during the experiment, the observed behaviour is a match with the outcome where the students were asked to order the modalities according to 
which one they liked the most; all of them preferred AV, then AV3D and finally FTF.

$\mathrm{AV}$ can be seen as inferior to $\mathrm{AV} 3 \mathrm{D}$ due to the need to convey instructions for the display of the clinical data set and confirmation of understanding by both parties. This was noted as a significant challenge by all participants. This does not occur with the FTF or AV3D modalities.

In AV3D learners had access to the real cast and to the 3D version. Some of them preferred to use the real cast as they felt more comfortable with it, but some showed interest in the 3D version, using it or manipulating it while waiting for the expert. 3D supported communication might require prior training to be effective and seen as a better user interface. Results, along with some of these observations, encourage further research into communication using AV3D modalities; particularly for distance courses for professionals who cannot afford to spend lengthy periods away from their surgeries and require greater convenience for the acquisition of continuing professional development. Although the number of participants is small, we believe similar findings could be obtained from testing a bigger group.

The authors gratefully acknowledge the seed-corn fund award from the University of Sheffield that enabled this project to take place. We are also indebted to Dr Angela Faircough, Dr Adrian K. Jowett and the fourth year BDS students from the 2010/11 cohort that selflessly assisted with this project.

1. Feeney L, Reynolds P A, Eaton K A, Harper J. A description of the new technologies used in transforming dental education. Br Dent J 2008; 204: 19-28.

2. BIS. Britain's superfast broadband future. Department for Business, Innovation and Skills. Online article available at http://www.culture.gov. uk/images/publications/10-1320-britains-superfast-broadband-future.pdf (accessed January 2012).

3. Downes P K. Putting it all together: dentistry and the internet. Br Dent J 2007; 203: 75-86.

4. Reynolds P A, Mason R, Eaton K A. Remember the days in the old school yard: from lectures to online learning. Br Dent J 2008; 204: 447-451.

5. Eaton K A, Reynolds P A, Grayden S K, Wilson N H. A vision of dental education in the third millennium. Br Dent J 2008; 205: 261-271.

6. Eaton K A, Reynolds P A. Continuing professional development and ICT: target practice. Br Dent J 2008; 205: 89-93.

7. Reynolds P A, Mason R, Harper J. The many faces of interaction. Br Dent J 2008; 204: 565-570.

8. Reynolds P A, Mason R, Eaton K A. Webcasting: casting the web more widely. Br Dent J 2008; 204: 145-149.

9. Mupparapu M. Voice over internet protocol for the orthodontic practice: a sensible switch from plain old telephone service. Am J Orthod Dentofacial Orthop 2008; 133: 470-475.

10. Cook J, Austen G, Stephens C. Videoconferencing: what are the benefits for dental practice? Br Dent J 2000; 188: 67-70.

11. Reynolds P A, Eaton K A, Mason R. Seeing is believing: dental education benefits from developments in videoconferencing. Br Dent J, 2008; 204: 87-92.

12. Miller K T, Hannum W M, Morley T, Proffit W R. Use of recorded interactive seminars in orthodontic distance education. Am J Orthod Dentofacial Orthop 2007; 132: 408-414

13. Bødker S. Applying activity theory to video analysis: how to make sense of video data in humancomputer interaction. In Nardi B A (ed) Context and consciousness: activity theory and human-computer interaction. pp 147-174. USA: Massachusetts Institute of Technology, 1996. 